\section{Pre-clinical anticancer drugs screening: perspectives from emerging models of glioma biology}

\section{Juan Sebastian Yakisich}

Dept. of Clinical Neuroscience, Karolinska Institute, Karolinska University Hospital, Sweden

The identification of putative cancer stem cells in tumors and cancer cell lines established the new dogma in the field of cancer that tumors are comprised of at least two different subpopulations of cells: cancer stem cells (CSCs ) and non-cancer stem cells (nonCSCs). ${ }^{1}$ Characterization of these subpopulations showed that CSCs are more resistant to anticancer drugs and are perhaps responsible for maintaining survival of cancer cells and for the failure of chemotherapy to obtain a cure., ${ }^{2,3}$ Thus, another new dogma has been established according to which eliminating the CSC subpopulation will eventually cure cancer. This has given rise to an upsurge of research aimed at finding ways to cure cancer by targeting CSCs.

It was initially proposed that elimination of the CSC subpopulation will leave the non-CSC fraction which will spontaneously stop dividing when these cells reach the Hayflick limit and eventually stop cancer growth. This assumption is wrong because if we assume that CSCs continuously originate non-CSCs, a fraction of newly originated non-CSCs (at the early stage of the Hayflick limit) will always be present with the potential to grow and produce symptomatic tumoral masses. ${ }^{4}$

Emerging models of gliomas are also diverging from the classical stem cell theory and their implications for anticancer drug screening may be extrapolated to other types of tumors. Gliomas are the most common brain tumor in adults and extensive research in glioma stem cells (GSCs) in cell lines and freshly isolated tumors has generated conflicting data originating alternative models of glioma biology. ${ }^{5}$ The simplistic classical model of CSCs and non-CSCs is an underestimation of the complexity of tumors and suggests that targeting only one cancer cell subpopulation will not be enough to cure cancer. The stemness phenotype model (SPM) proposes that all glioma cells have stem cell properties and the stemness of individual cells may vary from a pure GSC phenotype to a pure non-GSC phenotype, depending on specific microenviron- ments. These two phenotype extremes may interconvert to each other in permissive microenvironments. ${ }^{5}$ The complex system model (CSM), similar to the SPM, proposes that non-GSCs and GSCs can interconvert into each other. ${ }^{6} \mathrm{~A}$ third model, here called reprogramming model (RM), suggests that nonGSCs may reprogram into GSCs. ${ }^{7}$ Further experimental data support the concept that the phenotype of cancer cells, including the stemness, depends on the microenvironment: the regulation of CD133 by hypoxia, ${ }^{8}$ promotion of the stemness phenotype by acidic stress, ${ }^{9}$ maintenance of the stemness phenotype in the perivascular niche, ${ }^{10,11}$ and the differentiation of stem-like cells along tumor and endothelial lineages. ${ }^{12}$

Remarkably, all these new models above mentioned predict that to cure gliomas all cancer cells should be eliminated at once, otherwise any surviving cell will be able to generate a new tumor. In other words, development of therapies against putative GSCs will not be useful unless non-GSCs and cancer cells with intermediate phenotypes are targeted simultaneously. These findings related to glioma stem cells may be extrapolated to other types of tumors. Therefore, if we really want to cure cancer, we need to develop and put into clinical practice novel strategies or novel drugs or combinations of drugs that can eliminate $100 \%$ of cancer cells. The search for new therapies or drugs will also change the way we currently perform pre-clinical anticancer drug screening. In this regard, we need to develop or improve two main aspects of anticancer drug screening for the evaluation of anticancer compounds: i) novel proliferation assays and additional endpoint parameters able to monitor the ability of drugs to deplete $100 \%$ of cancer cells; and ii) novel animal models of cancers which can monitor tumor relapse after experimental treatment. The accomplishment of these goals at the pre-clinical level will allow more useful anticancer drugs to be selected and translated into advanced clinical trials.

\section{References}

1. Huntly BJ and Gilliland DG. Leukaemia stem cells and the evolution of cancerstem-cell research. Nat Rev Cancer 2005;5: 311-21.

2. Ropolo M, Daga A, Griffero F, et al. Comparative analysis of DNA repair in stem and nonstem glioma cell cultures. Mol Cancer Res 2009;7:383-92.
Correspondence: Juan Sebastian Yakisich, Dept. of Clinical Neuroscience R54, Karolinska University Hospital, Stockholm, Swedenl, S-141 86 , Huddinge, Sweden.

E-mail: sebastian.yakisich@ki.se

Acknowledgments: studies at author's laboratory are supported by grants from the Swedish Research Council and the Karolinska Institute.

Conflict of interest: the author reports no conflicts of interest.

Received for publication: 8 December 2010. Accepted for publication: 8 December 2010 .

This work is licensed under a Creative Commons Attribution 3.0 License (by-nc 3.0).

(C) Copyright J.S. Yakisich, 2011

Licensee PAGEPress, Italy

Drugs and Therapy Studies 2011; 1:e1

doi:10.4081/dts.2011.e1

3. Stubbs MC and Armstrong SA. Therapeutic implications of leukemia stem cell development. Clin Cancer Res 2007;13:3439-42.

4. Hanahan D and Weinberg RA. The hallmarks of cancer. Cell 2000;100:57-70.

5. Cruz M, Siden A, Tasat DR, Yakisich JS. Are all Glioma Cells Cancer Stem Cells? J Cancer Sci Ther 2010;2:100-6.

6. Laks DR, Visnyei $\mathrm{K}$ and Kornblum HI. Brain tumor stem cells as therapeutic targets in models of glioma. Yonsei Med J 2010;51:633-40.

7. Cheng L, Bao S and Rich JN. Potential therapeutic implications of cancer stem cells in glioblastoma. Biochem Pharmacol 2010;80:654-65.

8. Campos B and Herold-Mende CC. Insight into the complex regulation of CD133 in glioma. Int J Cancer 2011;128:501-10.

9. Hjelmeland $\mathrm{AB}, \mathrm{Wu} \mathrm{Q}$, Heddleston JM, et al. Acidic stress promotes a glioma stem cell phenotype. Cell Death Differ 2010;doi:10.1038/cdd.2010.150

10. Calabrese C, Poppleton H, Kocak M, et al. A perivascular niche for brain tumor stem cells. Cancer Cell 2007;11:69-82.

11. Charles N, Ozawa T, Squatrito M, et al. Perivascular Nitric Oxide Activates Notch Signaling and Promotes Stem-like Character in PDGF-Induced Glioma Cells. Cell Stem Cell 2010;6:141-52.

12. Wang R, Chadalavada K, Wilshire J, et al. Glioblastoma stem-like cells give rise to tumour endothelium. Nature 2010; in Press. 